\title{
ON THE SPHERE CONJECTURE OF BIRKHOFF
}

\author{
RICHARD JERRARD
}

(Communicated by George R. Sell)

\begin{abstract}
Birkhoff's sphere conjecture, now known to be false, says that if $f$ is a measure preserving homeomorphism of $S^{2}$ with the poles $N$ and $S$ fixed, and with no other periodic points, then $f$ is topologically conjugate to an irrational rotation of $S^{2}$. In this setting we say that $D \subset S^{2}$ is maximal for $f$ if $f(D) \cap D=\varnothing$ and $D$ is maximal with respect to that property. Also, $f$ is 2-small if for any circular ball $B$ such that $f(B) \cap B=\varnothing, f^{-1}(B) \cap f(B)=\varnothing$ also.

THEOREM. Any $f$ as above and also 2-small has a maximal set $D$ which is an open ball; its boundary contains $N$ and $S$ and is locally connected, and the area of $D$ is an irrational fraction of the area of $S^{2}$. This theorem gives another way of looking at the maps involved in the Birkhoff conjecture.
\end{abstract}

1. Introduction. We want to consider G. D. Birkhoff's sphere conjecture. It was one of sixteen questions raised in a paper presented by him in Chicago in September, 1941, which appeared only in summary form in Science [1].

BIRKHOFF'S CONJECTURE. If $f$ is an orientation and measure preserving homeomorphism of the two-sphere $S^{2}$ with exactly two fixed points but no other periodic points, then $f$ is topologically conjugate to an irrational rotation of $S^{2}$. We will call any such map a Birkhoff map.

A partial analysis of this problem was given by Montgomery [5]. However, the conjecture is now known to be false. One counterexample can be obtained by varying an example of a sphere mapping given by Handel [2] in 1982. More recently Markus [4] has shown that the conjecture is true for such a homeomorphism that is additionally the unit-time map for a smooth, conservative flow on $S^{2}$.

In this paper we obtain a result indicating in a different way how the idea of the conjecture can be interpreted. We use the following definition.

DEFINITION 1.1. Suppose that $f$ is a homeomorphism on a topological space $X$. A maximal set for $f$ is any connected, open set $D \subset X$ such that $f(D) \cap D=\varnothing$, and which is maximal with respect to that property. It then follows that $f^{-1}(D) \cap D=$ $\varnothing$ a'so.

For example, if $h$ is a rotation of the sphere, one maximal set is a lune from one fixed point to the other. We will show here that for many Birkhoff maps there exists a maximal set that is similar to such a lune. Furthermore, maximal sets are interesting in their own right. If we consider the iterates $f^{n}(D)$ of a maximal set $D$ we find that either they cover $S^{2}$ or $\operatorname{Bd}\left[\bigcup_{n} f^{n}(D)\right]$ contains a minimal set. The

Received by the editors August 5, 1986.

1980 Mathematics Subject Classification (1985 Revision). Primary 54H20.

Key words and phrases. Sphere homeomorphism, area preserving. 
nature of $D$ seems to depend crucially on whether its boundary is locally connected; both kinds of maximal sets may exist for the same function.

We want to make one further restriction on the maps studied here.

DEFINITION 1.2. A homeomorphism $f$ on $S^{2}$ is 2-small if for any circular ball $B$ such that $B \cap f(B)=\varnothing, f^{-1}(B) \cap f(B)=\varnothing$ also. Any rotation of the sphere of angle less than $2 \pi / 3$ is 2 -small. With this definition we can state the theorem about Birkhoff maps.

THEOREM 1.3. If $f$ is a 2-small orientation and measure preserving homeomorphism of $S^{2}$ which fixes the poles $N$ and $S$ and has no other periodic points, then $f$ has a maximal set $D$ which is an open ball. $\operatorname{Bd}(D)$ contains $N$ and $S$ and is locally connected, and the area of $D$ is an irrational fraction of the area of $S^{2}$.

2. Maximal sets. We first prove a few facts about maximal sets.

PROPOSITION 2.1. If $f$ is a homeomorphism on a Hausdorff space $X$, and is not the identity, then a maximal set for $f$ exists.

ProOF. We use the set $\mathbf{U}$ of all connected open sets such that if $U \in \mathbf{U}$ then $f(U) \cap U=\varnothing$. $\mathbf{U}$ is nonempty and is ordered by inclusion, and every chain in $\mathbf{U}$ has an upper bound, namely its union. Then by Zorn's Lemma $\mathbf{U}$ contains a maximal element $D$, and $D$ is a maximal set for $f$.

We will usually denote a maximal set by $D_{0}$, and put $D_{n}=f^{n}\left(D_{0}\right)$.

Proposition 2.2. Suppose that $D_{0}$ is a connected open set in a locally connected space $X$ such that $D_{0} \cap f\left(D_{0}\right)=\varnothing$. Then $D_{0}$ is a maximal set if and only if

$$
\operatorname{Bd}\left(D_{0}\right) \subset \operatorname{Bd}\left(D_{-1}\right) \cup \operatorname{Bd}\left(D_{1}\right) .
$$

ProOF. Assume that $D_{0}$ is a connected open set with $D_{0} \cap f\left(D_{0}\right)=\varnothing$, and that $x \in \operatorname{Bd}\left(D_{0}\right)$. First, if $x \notin \operatorname{Bd}\left(D_{-1}\right) \cup \operatorname{Bd}\left(D_{1}\right)$, there is a neighborhood $U$ of $x$ meeting neither $D_{-1}$ nor $D_{1}$ such that $f(U) \cap U=\varnothing\left[\right.$ if $f(x)=x$ then $x \in \operatorname{Bd}\left(D_{n}\right)$ for all $n]$. We see that

$$
\left(D_{0} \cup U\right) \cap f\left(D_{0} \cup U\right)=\left(D_{0} \cup U\right) \cap\left[f\left(D_{0}\right) \cup f(U)\right]=\varnothing
$$

because $D_{0} \cap f\left(D_{0}\right)=U \cap f(U)=\varnothing, U \cap f\left(D_{0}\right)=U \cap D_{1}=\varnothing$, and $D_{-1} \cap U=\varnothing$ implies $D_{0} \cap f(U)=\varnothing$. This property of $D_{0} \cup U$ shows that $D_{0}$ is not maximal, a contradiction.

Second, suppose that $\operatorname{Bd}\left(D_{0}\right) \subset \operatorname{Bd}\left(D_{-1}\right) \cup \operatorname{Bd}\left(D_{1}\right)$ but that $D_{0}$ is not maximal. Then $D_{0}$ is properly contained in some connected open $D_{0}^{\prime}$ and $D_{0}^{\prime} \cap f\left(D_{0}^{\prime}\right)=\varnothing$. There is then some $x \in \operatorname{Bd}\left(D_{0}\right) \cap D_{0}^{\prime}$. Now $x \in D_{0}^{\prime}$ implies $x \notin \bar{D}_{1}^{\prime}$ and $x \in \operatorname{Bd}\left(D_{0}\right)$ implies $x \in \bar{D}_{1} \subset \bar{D}_{1}^{\prime}$, a contradiction.

We next look at the behavior of the boundary points of a maximal set $D_{0}$.

DEFINITION 2.3. We put

$$
\begin{aligned}
B_{0} & =\left\{x \in \operatorname{Bd}\left(D_{0}\right) \mid x \notin \operatorname{Bd}\left(D_{1}\right)\right\} \\
B_{1} & =\left\{x \in \operatorname{Bd}\left(D_{0}\right) \mid x \notin \operatorname{Bd}\left(D_{-1}\right)\right\} \\
B_{p} & =\left\{x \in \operatorname{Bd}\left(D_{0}\right) \mid x \in \operatorname{Bd}\left(D_{-1}\right) \cap \operatorname{Bd}\left(D_{1}\right)\right\} \\
& =\operatorname{Bd}\left(D_{-1}\right) \cap \operatorname{Bd}\left(D_{0}\right) \cap \operatorname{Bd}\left(D_{1}\right) .
\end{aligned}
$$


In the example where $h$ is a small rotation of $S^{2}$ with $N$ and $S$ fixed, if the maximal set is a lune from $N$ to $S$ then $B_{p}=\{N, S\}, B_{1}$ and $B_{0}$ are the leading and trailing edges of the lune, $h\left(B_{0}\right)=B_{1}$, and $h\left(B_{p}\right)=B_{p}$. We shall see that this is a typical case. However, if $h$ is a rotation by $\pi$ radians, then $D_{0}$ is a hemisphere and $B_{p}$ is its entire great circle boundary. We will require $h$ to be 2 -small to rule out this situation.

EXAMPLE 2.4. Following Kerékjártó [3] we obtain a more disquieting example of a maximal set for the same 2-small rotation $h$ of $S^{2}$. Suppose that $S^{2}$ is described by the equation $x^{2}+y^{2}+z^{2}=1$ and that $\alpha:(0,1) \rightarrow S^{2}$ is a path that is asymptotic as $t \rightarrow 0(t \rightarrow 1)$ to the circle $z=-1 / 2(z=1 / 2)$, and which is strictly monotone increasing in its $z$ coordinate. Take $\beta$ to be the path obtained by following $\alpha$ by $h$. The two paths $\alpha$ and $\beta$ each encircle $S^{2}$ infinitely many times. Choose for $D_{0}$ the narrow open set enclosed by the images of $\alpha$ and $\beta$; then $B_{0}$ is the image of $\alpha, B_{1}$ is the image of $\beta$ and $B_{p}$ is the union of the two circles $z= \pm 1 / 2$. Again $h\left(B_{p}\right)=B_{p}$.

A maximal set of this sort gives no information about the map $h$ for $z>1 / 2$ or $z<-1 / 2$. Note that along the circular boundaries $\operatorname{Bd}\left(D_{0}\right)$ is not locally connected. The two kinds of maximal sets are fundamentally different.

Now suppose that $D_{0}$ is a maximal set for $f$ on $S^{2}$ and consider the sets $F=$ $\operatorname{Cl}\left[\bigcup\left\{D_{n} \mid n \in Z\right\}\right]$ and $G=S^{2}-F$. We show that $G$ is $f$-invariant.

PROPOSITION 2.5. $f(G)=G$.

ProOf. Suppose there exists $x \in f(G)$ such that $x \notin G$. Since $G$ is open there is a ball $B(x, \delta) \subset f(G)$ such that $B \cap G=\varnothing$. Now $B \cap D_{m} \neq \varnothing$ for some $m$, so $f^{-1}(B) \cap D_{m-1} \neq \varnothing$. But $f^{-1}(B) \subset G$ and $G \cap D_{m-1}=\varnothing$, and this contradiction shows that $f(G) \subset G$. In the same way it follows that $G \subset f(G)$.

\section{Proposition 2.6. $\mathrm{Bd}(G)$ contains a minimal set.}

ProOF. We need only note that $f(\operatorname{Bd}(G))=\operatorname{Bd}(G)$, and that $\operatorname{Bd}(G)$ is closed. Then any $x \in \operatorname{Bd}(G)$ generates a minimal set.

In Example 2.4 $G=\left\{(x, y, z) \in S^{2} \mid z<-1 / 2\right.$ or $\left.z>1 / 2\right\}$ and $\operatorname{Bd}(G)$ consists of the two circles $z= \pm 1 / 2$. Both circles are minimal sets if the rotation is irrational and contain finite minimal sets if it is rational. We want to find maximal sets where this situation does not occur. In the following theorem we construct maximal sets with locally connected boundaries.

THEOREM 2.7. If $f$ is a 2-small homeomorphism on $S^{2}$ other than the identity, ther there is a maximal set $D_{0}$ such that $\mathrm{Bd}\left(D_{0}\right)$ is locally connected, except possibly at fixed points of $f$.

PROOF. (a) We show that $D_{-1} \cap D_{1}=\varnothing$.

We will construct our maximal set $D_{0}$ for $f$ as a union of open sets

$$
D_{0}=\bigcup_{n}\left[B_{0 n} \cup R_{0 n}\right] \text {. }
$$

Each $B_{0 n}$ is a circular disc such that $B_{0 n} \cap f\left(B_{0 m}\right)=\varnothing$ for all $m \leq n$; because $f$ is 2 -small we have $f\left(B_{0 n}\right) \cap f^{-1}\left(B_{0 n}\right)=\varnothing$. The sets $R_{0 n}$ are chosen so that $R_{-1 n}$, $R_{0 n}$, and $R_{1 n}$ are disjoint. Therefore the maximal set $D_{0}$ will have the crucial property that $D_{-1}=\bigcup_{n} f^{-1}\left(B_{0 n} \cup R_{0 n}\right)$ and $D_{1}=\bigcup_{n} f\left(B_{0 n} \cup R_{0 n}\right)$ are disjoint. 
(b) $\operatorname{Bd}\left(D_{0}\right) \cap \operatorname{Bd}\left(D_{1}\right)$ is connected.

Suppose that $\operatorname{Bd}\left(D_{0}\right) \cap \operatorname{Bd}\left(D_{1}\right)$ is not connected. Then the connected set $\bar{D}_{0} \cup$ $\bar{D}_{1}$ separates $S^{2}$. Since $\operatorname{Bd}\left(D_{0}\right) \subset \operatorname{Bd}\left(D_{-1}\right) \cup \operatorname{Bd}\left(D_{1}\right)$, each component of $S^{2}-$ $\left(\bar{D}_{0} \cup \bar{D}_{1}\right)$ contains points of $D_{-1}$. But $D_{-1} \cap\left(\bar{D}_{0} \cup \bar{D}_{1}\right)=\varnothing$, so $D_{-1}$ is not connected, a contradiction.

(c) If $\operatorname{Bd}\left(D_{0}\right)$ is not locally connected at $x$, then $x \in B_{p}$.

First suppose that a maximal set $D_{0}$ such that $D_{-1} \cap D_{1}=\varnothing$ exists. We show how to alter $D_{0}$ so that if $\operatorname{Bd}\left(D_{0}\right)$ is not locally connected at $x$, then $x$ is a common boundary point of $D_{-1}, D_{0}$ and $D_{1}$, that is, $x \in B_{p}$. To see this, suppose that $x \in \operatorname{Bd}\left(D_{0}\right) \cap \operatorname{Bd}\left(D_{1}\right)$ but $x \notin \operatorname{Bd}\left(D_{-1}\right)$, and that $\operatorname{Bd}\left(D_{0}\right)$ is not locally connected at $x$. Choose a small open disc $B(x, \rho)$ not intersecting $\bar{D}_{-1}$. We first add to $D_{1}$ the set $A=B(x, \rho) \cap D_{0}$. This requires that $f(A)$ be added to $D_{2}$ (and hence subtracted from $D_{1}$ ) etc. We obtain $D_{1}^{\prime}=\left[D_{1} \cup A\right]-f(A)$ and $D_{0}^{\prime}=\left[D_{0} \cup f^{-1}(A)\right]-A$. Due to the subtraction of $A, D_{0}^{\prime}$ is no longer connected, but only one component of $\bar{D}_{0}^{\prime}$ meets $\bar{D}_{-1}$ for $\operatorname{Bd}\left(D_{0}\right) \cap \operatorname{Bd}\left(D_{-1}\right)$ is connected. The other components of $D_{0}^{\prime}$ are completely surrounded by $D_{1}$; we add all of their closures to $D_{1}^{\prime}$ to get the new $D_{1}$, with the corresponding new $D_{-1}$ and $D_{0}$. We have altered $D_{0}$ and $D_{1}$ so that the set within $B(x, \rho)$ where their common boundary was not locally connected is eliminated, and no new points where the boundary is not locally connected are created. A finite number of such operations (the ball $B(x, \rho)$ may be replaced by a topological ball) will produce the desired $D_{0}$. We will henceforth assume that if $\operatorname{Bd}\left(D_{0}\right)$ is not locally connected at $x$, then $x \in B_{p}$.

(d) Construction of $D_{0}$. We will obtain $D_{0}$ as a union of open sets which are constructed inductively. To begin, choose a point $P \in S^{2}$ such that $f(P) \neq P$, and denote by $B_{00}$ the maximal open disc with center at $P$ such that $B_{00} \cap B_{10}=\varnothing$ (in this notation the first subscript indicates how many times $f$ has been applied: $f\left(B_{00}\right)=B_{10}$; the second labels the inductive step). Note that because $f$ is a homeomorphism, the intersection $\bar{B}_{00} \cap \bar{B}_{10}$ corresponds exactly to $\bar{B}_{-10} \cap \bar{B}_{00}$. Now, if there is an open set $R_{00}$ bounded by $\bar{B}_{00} \cup \bar{B}_{10}$, and not containing $B_{-10}$ we unite $B_{00}$ with $R_{00}$ and define $D_{01}=\operatorname{Int}\left[\overline{B_{00} \cup R_{00}}\right]$, the first in the sequence of open sets $\left\{D_{0 n}\right\}$. The free boundary of $D_{01}$, namely $\operatorname{Bd}\left(D_{01}\right)-\left[\operatorname{Bd}\left(D_{-11}\right) \cup\right.$ $\left.\operatorname{Bd}\left(D_{11}\right)\right]$, consists of two circular $\operatorname{arcs} a_{01}$ and $b_{01}$. The open sets $D_{-11}, D_{01}$ and $D_{11}$ are pairwise disjoint, and $\operatorname{Bd}\left(D_{01}\right)$ consists of the two circular arcs plus points in $\operatorname{Bd}\left(D_{-11}\right) \cup \operatorname{Bd}\left(D_{11}\right)$.

The second step is typical (see Figure 1). We choose another point $P$ on the free boundary of $D_{01}$ equidistant from $D_{-11}$ and $D_{11}$ and such that this distance is maximal. The maximal open disc $B_{01}$, centered at $P$ and such that $\left(D_{01} \cup B_{01}\right) \cap$ $\left(D_{11} \cup B_{11}\right)=\varnothing$, is added to $D_{01}$ to get $D_{02}^{\prime}=D_{01} \cup B_{01}$. Note that $\bar{B}_{01}$ intersects both $\bar{D}_{-12}^{\prime}$ and $\bar{D}_{12}^{\prime}$, and if it intersects $\bar{B}_{11}$ then it must also intersect $\bar{B}_{-11}$. Now, if there is an open set $R_{01}$ bounded by $\bar{D}_{02}^{\prime} \cup \bar{D}_{12}^{\prime}$ (and not containing $D_{-12}^{\prime}$ ) we join it to $D_{02}^{\prime}$ to get the second set in the sequence, $D_{02}=\operatorname{Int}\left[\overline{D_{02}^{\prime} \cup R_{01}}\right]$. The free boundary of $D_{02}$ again consists of two circular arcs $a_{02}$ and $b_{02}$.

We can imagine that the disc $B_{01}$ grows from its center at $P$ until it strikes either the growing image disc $B_{11}$ or the fixed set $D_{11}$. The first possibility is illustrated in Figure 1. In the other case the growing $B_{01}$ must hit $D_{11}$ and $D_{-11}$ simultaneously (because $P$ is equidistant from these sets) and then the growing 


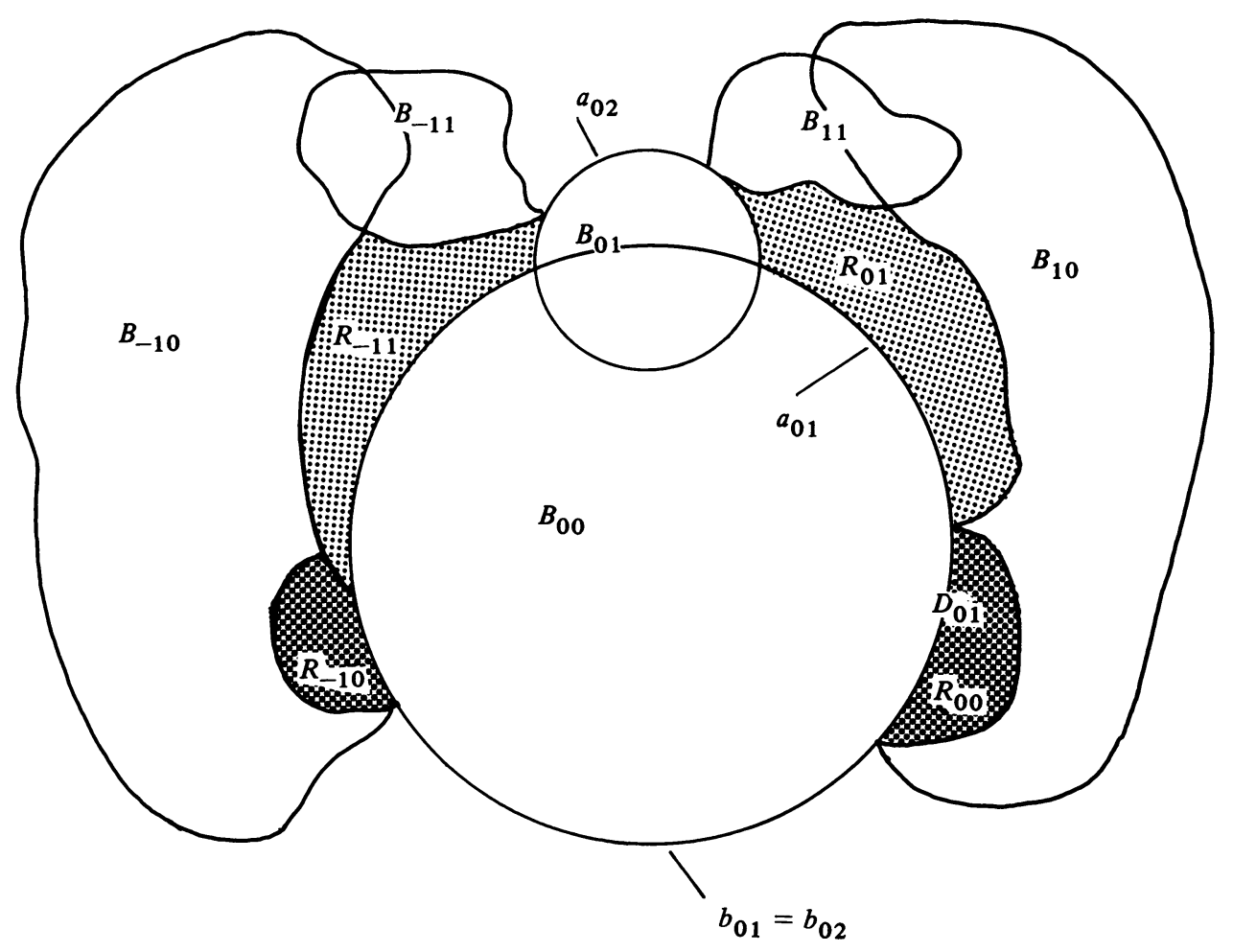

FIGURE 1. First stages in the construction of $D_{0}$

images $B_{-11}$ and $B_{11}$ will simultaneously hit $B_{00}$. If $P$ is on $a_{01}$ then $\bar{B}_{-11}$ and $\bar{B}_{11}$ will meet $\bar{B}_{00}$ on $b_{01}$. The result in both cases is that $a_{01}$ is buried and a new circular free boundary arc $a_{02}$ for $D_{02}$ is created, but that either all of $b_{01}$ or a subarc of it survives to become $b_{02}$.

The construction continues in this way to yield a sequence $\left\{D_{0 n}\right\}$ of open sets, each one contained in its successor. We define $D_{0}=\bigcup_{n} D_{0 n}$. This is clearly a connected open set such that $D_{0} \cap f\left(D_{0}\right)=\varnothing$. It is maximal because $\operatorname{Bd}\left(D_{0}\right) \subset$ $\left[\operatorname{Bd}\left(D_{-1}\right) \cup \operatorname{Bd}\left(D_{1}\right)\right]$. To see this first note that the sequence of radii of added discs converges to zero, for no added disc can have its center in a preceding disc, and the area of $S^{2}$ is finite. Then we can add no disc on $\operatorname{Bd}\left(D_{0}\right)$, so each point of $\operatorname{Bd}\left(D_{0}\right)$ must also be in $\operatorname{Bd}\left(D_{-1}\right) \cup \operatorname{Bd}\left(D_{1}\right)$. It follows from Proposition 2.2 that $D_{0}$ is a maximal set. This argument is unaffected if we add open discs $B$ and associated newly enclosed regions $R$ in some other order, which it will be necessary to do.

(e) $D_{0 m}$ cannot separate $S^{2}$.

At this stage we can see that if for some $m, D_{0 m}$ separates $S^{2}$, then $\operatorname{Bd}\left(D_{0}\right)$ is locally connected. For if $D_{0 m}$ separates $S^{2}$ so does $D_{0}$, and then $\operatorname{Bd}\left(D_{0}\right)$ has more than one component. Since $D_{-1}$ and $D_{1}$ are both connected, and since they both have boundary points in common with $D_{0}$, they must lie in different components of $\left(S^{2}-D_{0}\right)$. Then $D_{-1}, D_{0}$ and $D_{1}$ have no common boundary point, and $\operatorname{Bd}\left(D_{0}\right)$ must be locally connected. 
(f) Proof that $\mathrm{Bd}\left(D_{0}\right)$ is locally connected. In outline, the argument here goes as follows. We have seen that at each stage of the construction there are just two free circular arcs on the boundary on which discs may be added. We change the order of construction and add a complete sequence of discs $\left\{B_{0 n}\right\}$ continually on one of the arcs $a_{0 n}$, leaving $b_{0 n}$ possibly shortened but otherwise undisturbed. If this sequence $\left\{B_{0 n}\right\}$ converges to a point $x$ at which the boundary is not locally connected, and which is not a fixed point, then each member of the sequence $\left\{B_{1 n}\right\}$ must touch $b_{0 n}$; it must touch one of the two free arcs, and because $x$ is not a fixed point, $B_{1 n}$ is relatively far from $a_{0 n}$. It then follows that $f(x) \in b_{0 n}$, and as soon as we start adding discs on $b_{0 n}$ we arrive at a contradiction.

We first alter the construction by adding discs and regions in a different order. We first construct a sequence of discs $B_{0 n}$ with centers always on the free boundary arcs $a_{0 n}$, together with their associated regions $R_{0 n}$, to get the sequence $\left\{D_{0 n}\right\}$. We put $D_{0}^{\prime}=\bigcup_{n} D_{0 n}$. During this process we have $b_{01} \supset b_{02} \supset b_{03} \supset \cdots$. No disc is added with center on the second free boundary arc, though it may be shortened when $R_{0 n}$ is added; thus $b_{0 n}$ is a subarc of $b_{01}$. If during this process some added disc $B_{0 m}$ intersects $b_{0 m}$ then $D_{0 m+1}$ separates $S^{2}$ and we can apply the previous paragraph. $D_{0}^{\prime}$ has only one free boundary arc, as one has been eliminated by adding this first sequence of discs and regions. The single circular free boundary segment remaining in $D_{0}^{\prime}$ we will call $c_{00}$.

We next add successively to $D_{0}^{\prime}$ maximal discs $C_{0 n}$ centered on the free boundary $\operatorname{arcs} c_{0 n}$ together with the associated newly enclosed regions $S_{0 n}$ to get an increasing sequence of open sets $E_{0 n}$. Here $C_{0 n}$ and $S_{0 n}$ correspond to $B_{0 n}$ and $R_{0 n}$ in the earlier sequence. We put $E_{01}=D_{0}^{\prime} \cup \operatorname{Int}\left[\overline{C_{00} \cup S_{00}}\right]$, and $E_{0 n+1}=E_{0 n} \cup$ Int $\left[\overline{C_{0 n} \cup S_{0 n}}\right]$. The center of $C_{0 n}$ is at a point $P \in c_{0 n}$. Finally define $D_{0}=$ $D_{0}^{\prime} \cup\left[\bigcup_{n} E_{0 n}\right]$; as before, $D_{0}$ is a maximal set.

Now suppose that at some $y$ such that $f(y) \neq y, \operatorname{Bd}\left(D_{0}\right)$ is not locally connected. We will show first that either $y$ or $x=f^{-1}(y)$ is a limit point of a subsequence of one of the two sequences of added discs, and second that this fact leads to a contradiction.

For the first part, if $y$ is not a limit point of a sequence of added discs then it must be a limit point of a subsequence of a sequence of added regions, say of the sequence $\left\{R_{0 n}\right\}$. That is, each $\varepsilon$-ball centered at $y$ intersects infinitely many of the regions $\left\{R_{0 n}\right\}$. In particular it must intersect infinitely many of the boundaries $\left\{\operatorname{Bd}\left(R_{0 n}\right)\right\}$. But $\mathrm{Bd}\left(R_{0 n}\right)$ is a finite union of segments of disc boundaries, $\operatorname{Bd}\left(B_{0 n}\right)$ and $\operatorname{Bd}\left(B_{0 n-1}\right)$, and of homeomorphic images of disc boundaries, $f\left[\operatorname{Bd}\left(B_{0 n-1}\right)\right]$ and $f\left[\operatorname{Bd}\left(B_{0 n}\right)\right]$ (see $R_{01}$ in Figure 1). Therefore, either every neighborhood of $y$ intersects (and in fact contains) infinitely many discs $B_{0 n}$ or every neighborhood of $x=f^{-1}(y)$ does so. Thus we can conclude that either $x$ or $y$ is a limit point of a subsequence of one of the sequences of added discs.

For the second part, using the preceding paragraph we will assume that at some $x$ with $f(x) \neq x, \operatorname{Bd}\left(D_{0}\right)$ is not locally connected and every neighborhood of $x$ contains infinitely many of the added discs $\left\{B_{0 n}\right\}$ (we shall see presently that $x$ cannot be a limit point of the second sequence $\left\{C_{0 n}\right\}$ ). Recall that each disc $B_{0 n}$ is added to the free boundary segment $a_{0 n}$ and leaves the boundary segment $b_{0 n}$ undisturbed. Assume that the limit point $x$ is not on the free boundary segment $c_{00} \subset b_{0 n}$ and choose $N$ so that for $n>N$, $\operatorname{diam}\left(B_{0 n}\right)$ is much smaller than the 
distances $d\left[x, f^{-1}(x)\right]$ and $d[x, f(x)]$. Then for $n>N$, when $B_{0 n}$ is added we can imagine that it grows from its center $P \in a_{0 n}$ until it simultaneously strikes $D_{-1 n}$ and $D_{1 n}$. Far away, $B_{-1 n}$ and $B_{1 n}$ grow from their centers at $f^{-1}(P)$ and $f(P)$ and must both strike $D_{0 n}$ on its free boundary, and the only possibility is that they both strike $b_{0 n}$. We now have a sequence of discs converging to $x$ whose radii converge to zero. The image and counter-image sets all touch $b_{0 n}$ and since $f$ is uniformly continuous the diameters of the image and counter-image sets also converge to zero. Therefore $f^{-1}(x)$ and $f(x)$ both lie on $b_{01}$, one of the original circular boundary segments of $D_{01}$. Then because $b_{01} \subset \operatorname{Bd}\left(D_{01}\right)$ we find that $x \in \operatorname{Bd}\left(D_{11}\right)$ and $x \in \operatorname{Bd}\left(D_{-11}\right)$, or $x \in \bar{D}_{-11} \cap \bar{D}_{11}$.

Now, in continuing the construction of $D_{0}$ we add the first disc $C_{00}$ with center on $c_{00}$, together with the associated newly enclosed region $S_{00}$, to $D_{0}^{\prime}$ to obtain $E_{01}$. But each point of the free segment $c_{00}$ is now contained either in the interior of $E_{01}$ or in a locally connected circular boundary segment of $E_{01}$. The situation is entirely like that in Figure 1, where each point of $a_{01}$ lies either in the interior of $D_{02}$ or on the circular segment that is the common boundary of $D_{02}$ and $R_{-11}$. Therefore either $f(x) \in \operatorname{Int}\left(D_{0}\right)$, an impossibility because $x \in \operatorname{Bd}\left(D_{-1}\right)$, or $f(x)$ is on the common boundary of only two of the three sets $D_{-1}, D_{0}$ and $D_{1}$. This is again impossible because we have shown that $x \in B_{p}$.

This completes the argument which shows that if $D_{0}$ is constructed as above its boundary must be locally connected. It remains to clear up a couple of loose ends. First, the hypothetical point $x$ at which $\operatorname{Bd}\left(D_{0}\right)$ is not locally connected and which is the limit of a sequence of added discs cannot be the limit of the second sequence of added discs $\left\{C_{0 n}\right\}$. For during this stage in the construction there is only one free boundary segment $c_{0 n}$, and when $C_{0 n}$ is added on $c_{0 n}$ the corresponding $\operatorname{discs} \bar{C}_{-1 n}$ and $\bar{C}_{1 n}$ must intersect either $c_{0 n}$ or $\bar{C}_{0 n}$. Therefore, recalling that $P$ is the center of the added disc, as $n$ becomes large $\operatorname{diam}\left(C_{0 n}\right) \rightarrow 0$ and hence $d[P, f(P)] \rightarrow 0$ and $d\left[P, f^{-1}(P)\right] \rightarrow 0$. It follows that $x$ is then a fixed point of $f$ and is excluded from the argument by the hypothesis of the theorem. Second, we assumed after obtaining $x$ that it is not on $c_{00}$. If it is then we find as above that $x, f^{-1}(x)$ and $f(x)$ are all on $c_{00} \subset b_{01}$. But $x \in \operatorname{Bd}\left(D_{01}\right)$ implies $f(x) \in \operatorname{Bd}\left(D_{11}\right)$, and no point of $b_{01}$, by definition, lies on $\operatorname{Bd}\left(D_{11}\right)$.

3. Proof of Theorem 1.3. Using the results of $\S 2$ we can prove Theorem 1.3.

LEMMA 3.1. If $f$ is an area-preserving homeomorphism on $S^{2}$ (not the identity) then any maximal set $D_{0}$ for $f$ with locally connected boundary is an open disc.

Proof. Suppose that $D_{0}$ is not a disc, and therefore $D_{0}$ separates $S^{2}$. Its complement must contain exactly two components, for by Proposition 2.2 its boundary cannot contain more than two components; one of them lies in $\operatorname{Bd}\left(D_{-1}\right)$ and the other in $\operatorname{Bd}\left(D_{1}\right)$. Then $D_{1}$ lies in the component of the complement of $D_{0}$ which does not contain $D_{-1}$ and it also separates $S^{2}$. Similarly, $D_{n}$ lies in the component of the complement of $D_{n-1}$ that does not contain $D_{n-2}$. Thus the sets $D_{0}, D_{1}, D_{2}, \ldots$ are all disjoint from one another, and this is impossible for they all have the same area. 
We recall Definition 2.3 for the following lemma.

LEMMA 3.2. If $f$ is a 2-small area-preserving homeomorphism of $S^{2}$ (not the identity) and $D_{0}$ is a maximal set with locally connected boundary, then $f\left(B_{p}\right)=B_{p}$ and $f\left(B_{0}\right)=B_{1}$.

Proof. We first prove that $f\left(B_{p}\right)=B_{p}$. We assume that $x \in B_{p}$ and $f(x) \notin$ $\operatorname{Bd}\left(D_{-1}\right)$; thus $f(x) \in B_{1}$. Choose a small neighborhood $U$ of $f(x)$ so that $U \cap$ $\operatorname{Bd}\left(D_{0}\right) \subset B_{1}$. Then $U \cap \operatorname{Bd}\left(D_{0}\right)$ lies on the common boundary of the two open discs $D_{0}$ and $D_{1}$, and is locally connected because $x$ is not a fixed point. But $x \in \operatorname{Bd}\left(D_{1}\right)$ implies $f(x) \in \operatorname{Bd}\left(D_{2}\right)$ also, and $D_{0}, D_{1}$ and $D_{2}$ are pairwise disjoint because $f$ is 2-small. It follows that either $U \cap D_{0}$ or $U \cap D_{1}$ is not connected, say $U \cap D_{0}$. Then $D_{0} \cup\{f(x)\}$ separates $S^{2}$, with $D_{-1}$ and $D_{1}$ in different components. But the argument of Lemma 3.1 shows that this is not possible.

Now $f\left(B_{p}\right) \subset B_{p}$ and the same argument shows that $f^{-1}\left(B_{p}\right) \subset B_{p}$, or $B_{p} \subset$ $f\left(B_{p}\right)$; hence $B_{p}=f\left(B_{p}\right)$. It then follows that $f\left(B_{0}\right)=B_{1}$.

The following lemma completes the description of the maximal set for the 2-small Birkhoff map, except for the proof that its area is irrational. It consists of the open set between the two arcs $B_{0}$ and $B_{1}$, which run from $N$ to $S$ with $f\left(B_{0}\right)=B_{1}$.

LEMMA 3.3. If $f$ is a 2-small area-preserving homeomorphism of $S^{2}$ with fixed points at $N$ and $S$ as the only two periodic points, then any maximal set $D_{0}$ with locally connected boundary satisfies $B_{p}=\{N, S\}$.

ProOF. By Lemma $3.2, B_{p}$ is an invariant set. Since $D_{0}$ is an open disc, its locally connected boundary is a topological circle. We know that $\operatorname{Bd}\left(D_{0}\right)$ is the disjoint union of $B_{0}, B_{1}$ and $B_{p}$. Further $B_{0}$ (and hence $B_{1}$ ) is connected, for otherwise $D_{0} \cup D_{-1}$ would separate $D_{1}$. Thus $B_{0}$ and $B_{1}$ are open intervals in $\operatorname{Bd}\left(D_{0}\right)$ and are separated by $B_{p}$, which therefore has exactly two components. The two components of $B_{p}$ contain respectively the fixed points $N$ and $S$. Finally, each component of $B_{p}$ must consist of a single point: recalling that $B_{p}$ is the common boundary of the three open discs $D_{-1}, D_{0}$ and $D_{1}$, any component of $B_{p}$ which contains more than one point cannot be locally connected. But we have assumed that $\operatorname{Bd}\left(D_{0}\right)$ is locally connected (and 2.5 assures us that such maximal sets exist). Hence $B_{p}=\{N, S\}$.

PROOF OF THEOREM 1.3. By the preceding lemmas we know that there exists a maximal set $D_{0}$ for $f$ which is an open disc with the fixed points $N$ and $S$ on its locally connected boundary.

It remains to show that if $A_{S}$ is the area of $S^{2}$ and $A_{0}$ is the area of $D_{0}$, then $A_{0} / A_{S}$ is irrational. We assume that it is rational, that $A_{0} / A_{S}=p / q$, and consider the function $f^{q}$. This is also an area preserving homeomorphism of $S^{2}$ with the fixed points $N$ and $S$ as the only periodic points. It is also easy to see that if $f$ is 2 -small then so is $f^{q}$. We then know that if $f^{q} \neq 1$ there exists a maximal set $D$ for $f^{q}$ which is an open disc with $N$ and $S$ on its locally connected boundary, which in turn consists of two nonintersecting arcs from $N$ to $S$, one of which is the image under $f$ of the other. Furthermore the area of $D$ must be $q$ times the area of $A_{0}$, reduced modulo the area of $S^{2}$, that is, $q A_{0}\left(\bmod A_{S}\right)$. But $q A_{0}$ $\left(\bmod A_{S}\right)=q\left(p A_{S} / q\right)\left(\bmod A_{S}\right)=0$. Therefore $D$ does not exist and $f^{q}$ is the identity. Since we assumed only two periodic points for $f$ we conclude that $A_{0} / A_{S}$ is irrational. 


\section{REFERENCES}

1. G. D. Birkhoff, Some unsolved problems of theoretical dynamics, Science 94 (1941), 598-600.

2. Michael Handel, $A$ pathological $C^{\infty}$ diffeomorphism of the plane, Proc. Amer. Math. Soc. 86 (1982), 163-168.

3. B. V. Kerékjárt6, Vorlesungen uber Topologie. I, Grundlehren Math. Wiss., Bd. VII, SpringerVerlag, Berlin and New York, 1923, p. 195.

4. L. Markus, Three unresolved problems of dynamics, Preprint, University of Warwick, 1985.

5. D. Montgomery, Measure preserving homeomorphisms at fixed points, Bull. Amer. Math. Soc. 51 (1945), 949-953.

DEPARTMENT OF MATHEMATICS, UNIVERSity OF ILlinoIS, URBANA, ILlinOIS 61801 